\title{
Competência Social, Inclusão Escolar e Autismo: Um Estudo de Caso Comparativo
}

\author{
Síglia Pimentel Höher Camargo ${ }^{1}$ \\ Texas A\&M University \\ Cleonice Alves Bosa \\ Universidade Federal do Rio Grande do Sul
}

\begin{abstract}
RESUMO - A presente pesquisa analisou o perfil de competência social (CS) de uma criança pré-escolar com autismo, na escola comum comparado a uma criança com desenvolvimento típico e investigou a influência do ambiente escolar (sala de aula ou pátio) no perfil de CS de ambas. As interações sociais com seus pares foram filmadas, na escola, e a codificação dos vídeos foi realizada por um avaliador independente. Utilizou-se como instrumento a versão adaptada da Escala Q-sort de CS. Os resultados demonstraram que enquanto o perfil de competência social da criança com desenvolvimento típico pouco variou entre os contextos, a criança com autismo demonstrou maior frequência de comportamentos de cooperação e asserção social e menor frequência de agressão e desorganização do self, no pátio.
\end{abstract}

Palavras-chave: competência social; autismo; inclusão escolar; metodologia Q.

\section{Social Competence, School Inclusion and Autism: A Comparative Case Study}

\begin{abstract}
The aim of this study was to investigate the social competence ((SC) profile) of a child with autism in a regular in school, comparing it with a typically developing child and to investigate the influence of the school context (classroom or playground) on their SC profile. The social interactions with their peers were recorded at school and an independent judge coded the videos. The adapted version of the Q-Sort SC scale was used. The results demonstrated that while the SC profile of the typically developing child practically did not vary between contexts, the child with autism demonstrated a higher frequency of cooperation and social assertion behavior and a lower frequency of aggression and disorganization of the self in the playground.
\end{abstract}

Keywords: social competence; autism; school inclusion; Q methodology.

De acordo com o DSM-IV-TR (APA, 2002), o autismo caracteriza-se pelo comprometimento em três áreas do desenvolvimento: habilidades de interação social recíproca, de comunicação, e presença de comportamentos, interesses e atividades estereotipados. Diante dessas características é comum a crença na impossibilidade de permanência de crianças com autismo no ensino comum (Baptista, 2002). Entretanto, a eventual ausência de respostas das crianças com autismo deve-se muitas vezes à falta de compreensão do que está sendo exigido delas, ao invés de uma atitude de isolamento e recusa proposital (Bosa, 2002). Desse modo, a falta de investimento na criança com autismo, baseada na crença da total incapacidade para se comunicar e interagir pode levar à intensificação dos déficits inerentes às desordens do espectro do autismo (Bosa, 2002).

A literatura atualmente existente sobre autismo também enfatiza os prejuízos no desenvolvimento de suas habilidades interativas, onde o enfoque nas potencialidades dessas crianças é inexpressivo (Camargo \& Bosa, 2009). Todavia, para além das discussões sobre as limitações características do autismo, faz-se necessária a consideração da importância do ambiente no desenvolvimento dessas crianças.

1 Endereço para correspondência: UFRGS - Instituto de Psicologia. Rua Ramiro Barcellos, 2600, Sala 110. Bairro Santa Cecília - Porto Alegre, RS. CEP 90035-003. E-mail: sigliahoher@yahoo.com.br
O ser humano é um ser social em essência e o seu desenvolvimento atrela-se à qualidade das interações sociais que ele experimenta. As interações iniciais com pessoas significativas, em geral os pais e familiares, são o cerne do desenvolvimento social da criança. No entanto, a importância das interações para o desenvolvimento social da criança não se limita ao contexto familiar. A interação com pares é de extrema importância para o desenvolvimento infantil saudável, onde se desenvolvem formas específicas de cooperação, competição e intimidade (Hartup, 1992, 1996; Mathieson \& Banerjee, 2010).

A literatura aponta que a interação de crianças com seus pares proporciona resultados desenvolvimentais favoráveis (como a aquisição ou elaboração de habilidades sociais básicas de comunicação e cooperação), promove o autoconhecimento, o conhecimento do outro e proporciona o desenvolvimento cognitivo (Corsaro, 1997; Del Prette \& Del Prette, 2006; Hay, Payne, \& Chadwick, 2004; Pepler \& Craig, 1998; Williams, Ontai, \& Mastergeorge, 2010). Alguns autores enfatizam que esses ganhos podem ser obtidos através da educação infantil (Barbosa, 2007; Lordelo \& Carvalho, 1998; Oliveira \& Rosseti-Ferreira, 1993).

Por outro lado, diversas pesquisas apontam para uma variedade de resultados indesejáveis, resultantes da falta de amizades e oportunidades de interação com outras crianças (Girolametto \& Weitzman, 2007; Rubin, Coplan, \& Bowker, 
2009). Dessa forma, os companheiros representam uma fonte de relações imprescindíveis, provendo um contexto adicional único e poderoso que influencia as diferenças individuais durante o desenvolvimento social de qualquer criança (Castro, Melo, \& Silvares, 2003). Além disso, a competência social emerge nas interações com pares, sendo que a investigação dessas relações permite evidenciar diferenças individuais (Hartup, 1992; Chen \& French, 2008; Mathieson \& Banerjee, 2010).

A competência social espelha múltiplas facetas do funcionamento cognitivo, emocional e comportamental e é entendida como a capacidade de utilizar os recursos ambientais e pessoais para estabelecer um bom relacionamento, levando em consideração o contexto e o nível de desenvolvimento da criança (Chen \& French, 2008; Waters \& Sroufe, 1983). Trata-se, portanto, de um conjunto de comportamentos aprendidos no decurso das interações sociais com pares. Sendo assim, proporcionar às crianças com autismo oportunidades de conviver com outras crianças da mesma faixa etária possibilita o estímulo às suas capacidades interativas e o desenvolvimento da competência social, fornecendo modelos de interação e evitando o isolamento contínuo. A inclusão escolar de crianças com autismo surge como uma alternativa que pode fornecer esses contatos sociais e favorecer não só o seu desenvolvimento, mas o das outras crianças, na medida em que aprendem com as diferenças.

A proposta da inclusão supõe a participação de todos na escola regular, independente de seu talento, deficiência, origem socioeconômica ou cultural (Karagiannis, Stainback, \& Stainback, 1999; Kauffman, Landrum, Mock, Sayeski, \& Sayeski, 2005; Worrell, 2008) e, ao contrário da integração, supõe a adaptação da escola às necessidades e demandas de todas as crianças (Mantoan, 1998; Sassaki, 1998). Diversas são as evidências positivas de sua adequada implementação (Beyer, 2005; Mantoan, 1998; Sassaki, 1998; Zilmer, 2003), inclusive para crianças com autismo (Bosa, 2006; Serra, 2004; Yang, Wolfberg, Wu \& Hwu, 2003). Além das resistências e controvérsias oriundas de informações equivocadas e das poucas ações efetivas das políticas públicas para que a verdadeira inclusão se concretize, fato é que a inclusão escolar está prevista em lei (Brasil, 2001 - ECA, Lei nº 8.069 de 1990), é de extrema importância para o desenvolvimento de diversas crianças com transtornos do desenvolvimento e vem ocorrendo em diversas regiões do país. Dados publicados pelo Ministério da Educação e Secretaria de Educação Especial (http://portal.mec.gov.br/seesp) demonstram um aumento de $111 \%$ de estabelecimentos inclusivos em todo país, entre 2002 e 2005. Segundo o Censo Escolar 2006 (http://www. inep.gov.br), registrou-se $1,1 \%$ de crianças com autismo matriculadas em escolas regulares, número superior a 2005. Isso demonstra que o movimento da inclusão vem ganhando força e torna-se necessário e de fundamental importância o conhecimento fundamentado acerca das particularidades no desenvolvimento de crianças com autismo para a implementação de ações pedagógicas que respeitem e potencializem as habilidades que elas possuem.

Nos raros estudos encontrados na área da psicologia sobre a inclusão escolar de crianças com autismo, o foco é a percepção de pais e professores quanto a essa possibilidade e seus efeitos familiares e escolares (Avila, Tachibana,
\& Vaisberg, 2008; Goldberg, 2002; Kristen, Brandt, \& Connie, 2003; Lira, 2004; Sant'Ana, 2005). Desse modo, tais estudos não investigam aspectos diretamente relacionados à criança e suas interações, como contexto de expressão da competência social. Os estudos encontrados focando na inclusão escolar de crianças com autismo e suas interações buscaram avaliar programas de intervenção previamente estabelecidos sem utilizar uma metodologia de observação naturalística (Frederickson, Warren, \& Turner, 2005; Pereira, 2003), não envolveram amostras com crianças pré-escolares (Chamberlaim, 2002) e não consideraram a reciprocidade das interações numa perspectiva interacionista (Ochs, Kremer-Sadlick, Solomon, \& Sirota, 2001). Percebe-se, portanto, a carência de estudos relacionados à inclusão da criança com autismo, em escola regular de educação infantil, focando na interação dessa criança com as demais e na caracterização de suas possíveis potencialidades interativas.

Diante disso e dos diversos mitos acerca da educabilidade da criança com autismo, torna-se de grande relevância novas pesquisas no campo da psicologia, que demonstrem as potencialidades interativas dessas crianças no ensino regular e a influência do contexto no seu perfil de interação social. De acordo com Guaralnick, Connor, Hammond, Gottman e Kinnish (1996) a extensão em que as interações com pares são afetadas pelo ambiente social é uma área ainda pouco explorada, sobretudo em relação a crianças com desenvolvimento atípico. Um estudo conduzido por Kok, Kong e Bernard-Opitz (2002) comparou os efeitos do jogo estruturado do jogo facilitado na promoção de espontaneidade e responsividade na comunicação e comportamentos de jogo de crianças com autismo e encontrou melhores resultados em situação de jogo facilitado. No entanto, o estudo não conduziu as análises em um ambiente natural de sala de aula inclusiva e não explorou as diferenças de contexto em comparação a uma criança com desenvolvimento típico.

Diante do exposto, os objetivos deste estudo foram: 1) investigar o perfil de competência social de uma criança pré-escolar com autismo, em situação de inclusão escolar, comparado ao de uma criança com desenvolvimento típico, da mesma idade e sexo, 2) investigar a influência do ambiente escolar (sala de aula ou pátio) no perfil da competência social dessas crianças.

\section{Método}

\section{Participantes}

Para participar do estudo, tanto a criança com autismo (AU) quanto a criança com desenvolvimento típico (DT) deveriam ser da mesma faixa etária, sexo e grupo escolar. A ausência de deficiências fisicas ou sensoriais associadas ao autismo foi um critério de participação da criança com AU no estudo. A criança com DT, por sua vez, teve como critério de participação no estudo a ausência de intervenções terapêuticas e preocupações da escola ou da família sobre o seu desenvolvimento.

Considerando os critérios de inclusão, duas crianças pré-escolares do sexo masculino participaram do estudo. Uma 
criança tinha três anos e seis meses de idade e diagnóstico médico prévio de autismo (APA, 2002). A outra estava com quatro anos de idade e apresentava desenvolvimento típico.

A criança AU é uma criança ativa e com bom nível de desenvolvimento motor, sendo apta a correr, pular e mover-se independentemente. A linguagem é restrita e no momento da coleta de dados seu vocabulário era constituído por menos de 20 palavras espontâneas. Apresenta ecolalia e alguns comportamentos estereotipados (ex. balanço do corpo e movimentos repetitivos da cabeça). Ambos os pais possuem curso superior completo e são divorciados. Possui uma irmã de cinco anos, que estuda na mesma escola. A criança com desenvolvimento típico (DT) é filho único, sendo que ambos os pais possuem curso superior completo e também são divorciados. As duas crianças frequentavam regularmente a mesma classe da escola regular, na rede de ensino privado de Porto Alegre/RS. Elas frequentavam o turno integral da escola, inclusive realizando ali suas refeições.

\section{Instrumentos e Materiais}

Foi utilizada a versão adaptada da Escala Q-sort de Competência Social (Almeida, 1997). Esse instrumento foi construído com base na Metodologia Q introduzida por Willian Stephenson em 1953. Trata-se de um instrumento de medida ideográfico que permite avaliar as diferenças e semelhanças individuais a partir da identificação de padrões de organização do comportamento social, levando em conta os fatores de contexto e de desenvolvimento. Esse instrumento é originalmente composto por 53 itens. Entretanto, o mesmo foi adaptado para o português brasileiro e para a realidade da criança com autismo, sendo que um manual de definições operacionais dos itens foi desenvolvido. A versão final consta de 46 itens, cuja acurácia foi concluída com base em um estudo piloto desenvolvido com dois avaliadores "cegos" aos objetivos do estudo. Os itens pertencem a diferentes sub-escalas que se referem a cinco dimensões da competência social: Sociabilidade/cooperação (8 itens), Asserção Social (8 itens), Agressão (11 itens), Dependência (11 itens), Desorganização do Self (oito itens). Na versão adaptada da Escala $Q$-sort de competência social, os 46 itens devem ser distribuídos em um continuum de categorias e pontuações que variam de 1 a 7 por ordem de representatividade (ou de significância baseado na ocorrência do comportamento descrito no item). Assim, os itens mais característicos ou que melhor descrevem a criança são posicionados no extremo direito do continuum e têm, portanto, uma pontuação mais alta, enquanto os menos característicos têm pontuações mais baixas, no outro extremo. A categoria 4, que ocupa a posição central da escala é não representativa para a descrição da pessoa, isto é, estes itens têm uma significação (e pontuação) neutra nessa descrição. A definição de cada pontuação da escala, reflete a frequência ou intensidade com que o item ocorreu, sendo que: 1- o item descrito no cartão ocorre muito raramente, 2- raramente, 3- ocasionalmente, 4- não ocorre, 5- pouco frequentemente, 6- frequentemente e 7- muito frequentemente. A distribuição final dos itens deve ajustar-se a uma distribuição simétrica, contendo obrigatoriamente quatro itens nas categorias $1 \mathrm{e}$ 7 do continuum, seis itens nas categorias 2 e 6 , oito itens nas categorias 3 e 5 e 10 itens na categoria 4. Desse modo, os itens que se encontram nos extremos da escala são os "pouco" e "bastante" representativos da criança observada. Devido à redução do número de itens e da tênue variação entre as pontuações da escala, as categorias 1-2 e 6-7 foram consideradas extremos da escala.

\section{Procedimentos e considerações éticas}

Para realizar o estudo, uma escola privada de educação infantil foi selecionada por critério de conveniência, ou seja, essa escola possuía um aluno com AU que apresentou os critérios de participação estabelecidos para este estudo. O sexo masculino foi selecionado por apresentar maior incidência de autismo, e a idade por contemplar um período em que as crianças passam a experienciar contatos sociais mais amplos que a família, com os pares, através da educação infantil. Uma vez selecionada a escola, essa foi contatada para esclarecimentos quanto à natureza e objetivos do estudo. Após o consentimento da instituição, a criança com DT foi selecionada com base em um sorteio entre as demais crianças com desenvolvimento típico da mesma classe da criança com AU. Foram contatados os pais ou responsáveis pelas crianças para esclarecimentos necessários e preenchimento da ficha de dados sociodemográficos e do Termo de Consentimento Livre e Esclarecido. O projeto foi submetido ao Comitê de Ética do Instituto de Psicologia da UFRGS, do qual recebeu aprovação.

A observação sistemática da criança com AU e da criança com DT com seus pares, foi realizada em dois contextos: sala de aula e pátio. Para tanto, cada criança foi filmada por um período de uma hora, sendo 30 minutos em cada contexto. Para isso, foi utilizada uma câmera de vídeo móvel que permitiu focar o ambiente e a criança de forma mais ampla e direta possível. As filmagens foram conduzidas pela primeira autora e uma aluna de graduação do Curso de Psicologia da UFRGS, treinada para os procedimentos do estudo.

Com o intuito de gerar uma amostragem de comportamentos e evitar a influência de possíveis condições (ex: fadiga, sono, indisposição, etc.) em um único dia, as filmagens das duas crianças foram conduzidas em dias diferentes, uma vez por semana, em três semanas distintas. Perídos de 20 minutos de filmagem em cada dia foram subdivididos, sendo aleatoriamente selecionados trechos de 10 minutos. A partir disso, obteve-se o período de 30 minutos para cada contexto com três sessões de 10 minutos aleatoriamente selecionadas, realizadas em três semanas distintas. Foram realizadas visitas prévias à coleta de dados, à sala de aula dos participantes, a fim de promover a familiarização das crianças e seus colegas com a presença dos pesquisadores.

\section{Procedimento de Registro e Análise dos Dados}

As filmagens realizadas para cada criança foram codificadas e registradas através da versão adaptada da Escala Q-Sort de Competência Social pelo mesmo avaliador, psicólogo, "cego" aos objetivos do estudo e ao diagnóstico da criança com autismo. O avaliador foi treinado e instruído 
a assistir separadamente as filmagens de cada criança, em cada contexto, e distribuir os itens nas categorias da escala Q-Sort de acordo com o seu julgamento quanto à representatividade dos mesmos, ao longo do continuum de sete pontos. Visando minimizar o enviesamento dos dados, os vídeos foram apresentados ao avaliador alternadamente, de forma a contrabalançar as possíveis influências da sequência e do contexto assistido e evitar a comparação imediata das duas crianças no mesmo contexto ou da mesma criança nos dois contextos. Desse modo, o avaliador assistiu ao vídeo da criança com AU no pátio, seguido pelo vídeo da criança com DT na sala de aula e depois no pátio e, novamente, a criança com AU na sala de aula. Após a distribuição final dos itens para cada criança e contexto nas categorias do continuum, a pontuação atribuída para cada item foi anotada em uma folha de registro de dados, onde os itens estavam distribuídos em suas respectivas dimensões da escala para facilitar as análises posteriores. A partir da análise estatística descritiva, as diferenças e semelhanças entre os perfis de interação identificados para cada criança e entre os diferentes contextos da interação (pátio e sala de aula) foram examinadas.

\section{Resultados e Discussão}

Os resultados obtidos serão apresentados comparando-se, inicialmente, o perfil de competência social da criança com AU e da criança com DT nos dois contextos escolares. Posteriormente, o foco da discussão centra-se na análise da influência do contexto no perfil de competência social de cada criança. Para facilitar a compreensão, as diferenças e semelhanças entre as crianças e os contextos serão apresentadas de acordo com as cinco dimensões que compõem a Escala Q-Sort de Competência Social (Almeida, 1997). Foram considerados semelhantes aqueles comportamentos/itens que obtiveram o mesmo escore ou escores com no máximo um ponto de diferença (ex. 2 e 3), incluindo os pares 0 e 0 (que é a representação do escore 4 - o comportamento não ocorre) e 3 e 5 (uma vez que o 4 é pontuado como 0). Acima disso, os comportamentos foram considerados diferentes.

\section{Análise do perfil de competência social da criança com AU x criança com DT}

Os resultados obtidos neste estudo indicaram diferenças, mas também algumas semelhanças entre as duas crianças nos dois contextos investigados. Analisando-se as diferenças entre ambas nas dimensões de Socialibilidade/Cooperação e Asserção Social (Tabela 1), pode-se perceber que os itens nos quais o perfil de interação da criança com AU se diferenciou da criança com DT no pátio referem-se às dificuldades sociais e de interação características do espectro do autismo, amplamente mencionadas pela literatura (APA, DSM IV-TR, 2002; Bosa, 2006; Facion, Marinho, \& Rebelo, 2002; Kanner, 1943; Klin, 2006).

Desse modo, não se revelaram surpreendentes os escores mais baixos da criança com AU nos itens 1, 2, 9 e 21 (dimensão Sociabilidade/Cooperação) e 6, 31, 37 e 44 (dimensão Asserção Social), uma vez que esses se referem especificamente às habilidades que se encontram prejudicadas nesta condição, tais como: habilidade de integração grupal, disposição interna para colaborar e ajudar os outros e manter-se motivada nas tarefas, bem como habilidades para manter o foco de atenção e para a expressão de desejos, sentimentos e opiniões. Alguns estudos internacionais, comparativos entre crianças com AU e crianças com DT (Anderson, Moore, Godfrey \& Fletcher-Flinn, 2004), também demonstraram que os dois grupos diferiram significativamente em atividades coletivas, nos mesmos ambientes.

Na metade dos itens das dimensões de Sociabilidade/Cooperação (itens 3, 7, 33 e 42) e Asserção Social (itens 17, 36, 40, 43), a criança com AU apresentou um perfil de interação semelhante à criança com DT. Isso indica que, ao contrário do que muitos mitos em relação ao autismo podem sugerir, as dificuldades características da síndrome não impedem completamente a criança com AU de apresentar comportamentos de sociabilidade e asserção social, tal como uma criança com DT. O estudo desenvolvido por Guaralnick et al. (1996) com crianças com transtornos da comunicação também encontrou diferenças e semelhanças dessas em relação a crianças com DT, nos mesmos ambientes. Desse modo, o fato de a criança com AU ser solicitada pelo grupo, mesmo que com pouca frequência (item 3), e aceitar as sugestões e pedidos do mesmo (item 7) tal como a criança com DT, sugere que ela pode ser incluída, aceita e considerada pelos companheiros. Isso reforça a literatura a esse respeito (Karagiannis, Stainback, \& Stainback, 1999), que enfatiza os ganhos com a inclusão escolar não só para criança com autismo, quanto para as demais. Além disso, os teóricos da interação social (Hartup, 1996; Pepler \& Craig, 1998) também são unânimes em reiterar a importância das interações com pares para o desenvolvimento da competência social de todas as crianças. Fortalecendo essa noção está ainda o resultado indicando que a criança com AU mostrou-se frequentemente amigável com os colegas (item 42), demonstrando afetividade (abraçando ou beijando o colega). Esse resultado é corroborado por estudos que já apontavam que nem todas as crianças com AU possuem "aversão ao toque físico" (Klin, 2006; Trevarthen, 2000). Além disso, tal como a criança com DT, a criança com AU frequentemente transparece boa disposição (sorri e faz graça- item 36) mesmo diante de incidentes (por exemplo, rir de si própria ao cair, jogando bola).

A criança com AU ocasionalmente se mostrou incapaz de aguardar a sua vez numa situação de interação (item 17). Contudo, chama-se a atenção para o fato de que esse comportamento também ocorreu com a criança com DT na mesma frequência, não sendo portanto, uma característica específica do autismo. Situação semelhante ocorreu quanto à capacidade de ceder aos argumentos do grupo (item 33), em que a criança com DT também se mostrou passiva e submissa com frequência. Entretanto, passividade e submissão parecem ser características dessa criança com DT.

No contexto de sala de aula, as diferenças entre as crianças na dimensão sociabilidade/cooperação ocorreram em dois itens em que elas assemelharam-se, no pátio (itens 3 e 7). Isso pode ser explicado pela influência do contexto no comportamento social da criança, o qual será discutido mais adiante.

Conforme mencionado, a tríade sintomatológica dos transtornos do espectro do autismo envolve comprometimen- 
Tabela 1. Escore da Escala de Competência Social da criança com autismo (AU) e da criança com desenvolvimento tipico (DT) no pátio (P) e sala de aula (S) nas dimensões de sociabiliadade/cooperação, asserção social e desorganização do self

\begin{tabular}{|c|c|c|c|c|c|c|c|c|c|c|c|c|c|c|}
\hline \multicolumn{5}{|c|}{ Sociabilidade/cooperação } & \multicolumn{5}{|c|}{ Asserção social } & \multicolumn{5}{|c|}{ Desorganização do self } \\
\hline \multirow{2}{*}{ Item $^{(a)}$} & \multicolumn{2}{|c|}{ AU } & \multicolumn{2}{|c|}{ DT } & \multirow{2}{*}{ Item $^{(b)}$} & \multicolumn{2}{|c|}{$\mathrm{AU}$} & \multicolumn{2}{|c|}{ DT } & \multirow{2}{*}{ Item $^{(\mathrm{c})}$} & \multicolumn{2}{|c|}{$\mathrm{AU}$} & \multicolumn{2}{|c|}{ DT } \\
\hline & $\mathrm{P}$ & $\mathrm{S}$ & $\mathrm{P}$ & $\mathrm{S}$ & & $\mathrm{P}$ & $\mathrm{S}$ & $\mathrm{P}$ & $\mathrm{S}$ & & $\mathrm{P}$ & $\mathrm{S}$ & $\mathrm{P}$ & $\mathrm{S}$ \\
\hline 1 & 2 & 1 & 6 & 6 & 6 & 3 & 1 & 7 & 7 & 15 & 5 & 5 & 1 & 1 \\
\hline 2 & 2 & 2 & 5 & 6 & 17 & 3 & 3 & 3 & 5 & 16 & 3 & 7 & 0 & 0 \\
\hline 3 & 5 & 2 & 5 & 5 & 31 & 1 & 2 & 6 & 5 & 24 & 3 & 3 & 3 & 2 \\
\hline 7 & 3 & 3 & 5 & 6 & 36 & 5 & 3 & 6 & 6 & $25^{(\mathrm{d})}$ & 0 & 3 & 0 & 0 \\
\hline $9^{\text {(d) }}$ & 0 & 0 & 3 & 0 & 37 & 5 & 0 & 7 & 7 & 26 & 5 & 3 & 0 & 0 \\
\hline 21 & 1 & 1 & 6 & 3 & 40 & 6 & 5 & 6 & 5 & 27 & 5 & 5 & 1 & 1 \\
\hline 33 & 6 & 6 & 5 & 5 & 43 & 3 & 5 & 5 & 6 & 41 & 1 & 0 & 2 & 3 \\
\hline 42 & 6 & 5 & 7 & 6 & 44 & 2 & 2 & 6 & 7 & 46 & 0 & 3 & 2 & 1 \\
\hline
\end{tabular}

(a) 1. Integra-se facilmente no grupo, 2. Colabora e dispõe-se a ajudar, 3. Os outros perguntam-lhe ou pedem a sua opinião, 7. Aceita as sugestões /opiniões/ pedidos dos companheiros, 9. A criança tenta apaziguar e conciliar os colegas, 21. Canaliza a atenção do grupo para a tarefa, 33 . É capaz de ceder perante argumentos convincentes do grupo, 42. Mostra-se amigável.

(b) 6. Age com independência e demonstra autonomia relativa ao grupo,17. Fala e age com ponderação,31. Expressa com desenvoltura os seus desejos e sentimentos, 36. Reage de forma divertida e com graça, 37. Expressa as suas intenções de modo claro e direto, 40. Mantém a calma em momentos de tensão ou conflito, 43. Tem boas ideias para fazer as coisas, 44. Mostra-se determinada e motivada.

(c) 15. Faz propostas que são ignoradas pelo grupo, 16. A criança é objeto de rejeição ativa pelo grupo, 24. Mostra-se ansiosa na situação de grupo, 5 . Apresenta reações negativas quando contrariada, 26. Ri sem propósito e faz brincadeiras sem sentido, 27. Chama a atenção para assuntos irrelevantes e distrai o grupo, 41. Reage mal com chamadas de atenção ou críticas, 46. Irrita-se facilmente e zanga-se.

(d) Estes foram os únicos itens em que o escore zero para as duas crianças ou contextos não foi como semelhança. A pontuação pode tanto indicar que o evento não ocorreu, para que o comportamento pudesse ser avaliado, quanto ocorreu, mas o comportamento não foi observado.

tos qualitativos nas áreas de interação social, comunicação e comportamento. Considerando esse aspecto e o fato de que a dimensão desorganização do self (Tabela 1) basicamente caracteriza as dificuldades do controle emocional e a ruptura com a situação social, é possível entender os escores mais altos obtidos pela criança com AU em relação à criança com DT, nos dois contextos. Entretanto, dentre os itens em que ambas diferenciam-se no pátio, com uma maior pontuação atribuida à criança com $\mathrm{AU}$, essa pontuação reflete uma eventual frequência do comportamento descrito no item. No item 16, por exemplo, a criança com AU foi objeto de rejeição ativa pelo grupo apenas ocasionalmente. Além disso, no item 46 ela não apresentou comportamentos de irritar-se e zangar-se facilmente e este, mesmo passando a ocorrer na sala de aula com mais frequência que a criança com DT, também é apenas casual. Essa evidência ajuda a repensar a prevalente noção de que essas crianças irritam-se e zangam-se facilmente com qualquer tentativa de aproximação, desencadeando crises de agressividade e comportamentos auto-lesivos. Tais comportamentos, quando ocorrem, estão em geral associados a uma situação de protesto a uma exigência ou demanda a que não conseguem corresponder e têm uma função comunicativa. Levando em consideração o conceito de competência social numa perspectiva organizacional e relacional (Hartup, 1992, 1996; Waters \& Sroufe, 1983) é possível afirmar que antes de um comportamento de agressão, isso reflete uma solução adaptativa da criança de acordo com o seu nível de desenvolvimento e limitações de comunicação, mas que permite indicar suas necessidades. Da mesma forma, comportamentos de irritabilidade (choro, gritos, agitação) podem ser explicados por uma tentativa de comunicação e protestos a mudanças súbitas de atividades, as quais desorganizam o comportamento dessas crianças que tendem a apresentar uma adesão inflexível a rotinas (Bosa, 2006).
A apresentação de reações negativas quando contrariada (item 25) também não foi um comportamento frequentemente observado na criança com $\mathrm{AU}$, o qual não ocorreu no pátio e apareceu ocasionalmente na sala de aula. Por outro lado, mostrar-se ansiosa na situação do grupo (item 24) não representa uma característica exclusiva da criança que possui dificuldades nas interações sociais, sendo esse um comportamento que ocorreu nas duas crianças, em ambos os contextos. A presença de apenas um item que foi semelhante na sala de aula (item 24), bem como o maior número de itens que se mostraram diferentes entre as crianças (e com frequência maior que o pátio para a criança com autismo), também pode ser explicada pelas características específicas de cada contexto e a sua influência no perfil de interação das crianças.

Na dimensão Agressão (Tabela 2), os dados novamente corroboram a inadequação de crenças que inviabilizam a inserção da criança com AU no ensino comum. De acordo com Baptista, Vasques e Rublescki (2003), muitos educadores resistem ao trabalho com crianças autistas devido a temores em não saber lidar com a agressividade de tais crianças. Entretanto, essa não é uma característica prototípica da síndrome, sendo também comum em crianças com desenvolvimento típico. Desse modo, os resultados demonstram que a criança com AU, assim como a criança com DT, não apresentou a maioria dos itens referentes a aspectos dominadores, nos dois contextos. Além disso, naqueles comportamentos em que a criança com AU obteve uma pontuação diferente e maior que a criança com DT, refletem-se as dificuldades inerentes ao autismo. No pátio, a criança com AU apresentou escore maior que a outra criança quanto a "não ouvir e nem se dispor a negociar" (item 28). Esse foi um comportamento frequentemente observado e que pode estar associado à sua dificuldade em iniciar ou manter uma conversação, assim como em compreender a situação social. Do mesmo modo, o outro item em que obteve escore maior 
Tabela 2. Escore da Escala de Competência Social da criança com autismo (AU) e da criança com desenvolvimento tipico (DT) no pátio (P) e sala de aula (S) nas dimensões agressão e dependência

\begin{tabular}{|c|c|c|c|c|c|c|c|c|c|}
\hline \multicolumn{5}{|c|}{ Agressão } & & \multicolumn{4}{|c|}{ Dependência } \\
\hline \multirow{2}{*}{ Item $^{(a)}$} & \multicolumn{2}{|c|}{$\mathrm{AU}$} & \multicolumn{2}{|c|}{ DT } & \multirow{2}{*}{ Item $^{(b)}$} & \multicolumn{2}{|c|}{$\mathrm{AU}$} & \multicolumn{2}{|c|}{ DT } \\
\hline & $\mathrm{P}$ & $\mathrm{S}$ & $\mathrm{P}$ & $\mathrm{S}$ & & $\mathrm{P}$ & $\mathrm{S}$ & $\mathrm{P}$ & $\mathrm{S}$ \\
\hline 4 & 6 & 6 & 5 & 3 & 8 & 6 & 7 & 3 & 2 \\
\hline 5 & 3 & 2 & 2 & 2 & 10 & 5 & 5 & 5 & 3 \\
\hline 11 & 0 & 0 & 0 & 0 & 20 & 0 & 0 & 3 & 3 \\
\hline 12 & 0 & 0 & 0 & 0 & 22 & 3 & 5 & 3 & 3 \\
\hline 13 & 0 & 0 & 0 & 0 & 29 & 7 & 7 & 1 & 2 \\
\hline 14 & 0 & 3 & 0 & 0 & 30 & 6 & 7 & 1 & 3 \\
\hline 18 & 2 & 6 & 0 & 1 & 32 & 7 & 6 & 5 & 3 \\
\hline 19 & 0 & 0 & 0 & 0 & 34 & 2 & 2 & 3 & 5 \\
\hline 23 & 1 & 1 & 7 & 7 & 35 & 2 & 0 & 2 & 5 \\
\hline 28 & 7 & 6 & 2 & 2 & 38 & 5 & 6 & 2 & 2 \\
\hline 39 & 0 & 0 & 0 & 0 & 45 & 7 & 5 & 3 & 5 \\
\hline
\end{tabular}

(a) 4. Não partilha o espaço com os outros e tende a se impor, 5. Não deixa que os outros dêem sugestões, 11. Gosta de irritar e provocar os companheiros, 12. É agressiva verbalmente: fala nomes, deprecia, grita, etc.., 13. É agressiva fisicamente: empurra, agarra, belisca, bate..., 14. É impulsiva: perturba ou interrompe os outros com frequência, 18. Não ouve os outros nem se dispõe a negociar, 19. Tem um comportamento de oposição: protesta, resiste a ceder, discorda..., 23. É diretiva: demonstra formeza e determinação, 28. Dificuldade em iniciar, dirigir ou manter a atenção em uma atividade em grupo..., 39. Tende a manipular para conseguir o que deseja.

(b) 8. Precisa que os outros lhe digam o que fazer, 10. Criança mostra-se ambivalente , 20. Muda de opinião com frequência, 22. Mostra dificuldades em tomar decisões, 29. Não se envolve na atividade do grupo: participa pouco e tende a isolar-se, 30. Mantém-se distraída: parece ausente, como se sonhasse acordada, 32. Não toma posição nos impasses ou desacordos, 34. Sensibilidade aos sinais de rejeição dos outros, 35 . Receia contrariar o grupo e não exprime opiniões contrárias aos companheiros, 38. Age com timidez, mostra dificuldade em relacionar-se com os outros, 45 . Obedece às ordens sem questionar.

que a criança com DT (item 28), demonstra a dificuldade em iniciar, dirigir ou manter a atenção por mais de alguns minutos, típica de crianças nessa condição. Na sala de aula, apesar de apresentar maiores dificuldades em relação a essa dimensão, os comportamentos não deixam de refletir as características do autismo já mencionadas.

Na dimensão Dependência (Tabela 2), a criança com AU apresentou um perfil de interação com características de maior dependência que a criança com DT, tanto no pátio quanto na sala de aula. Porém, isso foi mais evidente na sala de aula, indicando a maior necessidade de auxílio da criança com autismo nesse contexto. A maioria dos itens de Dependência que a criança com AU apresentou com maior frequência, novamente estão relacionados a situações que abarcam os comprometimentos da síndrome, inevitavelmente aumentando os escores da mesma. Porém, apesar de ser maioria, não foram em todos os itens que a criança com AU mostrou-se mais dependente, sobretudo no pátio. Apesar de pouco frequente como a criança com $\mathrm{AU}$, a criança com DT mostrou-se ambivalente (item 10), tendendo a solicitar os outros de forma negativa (opondo-se, não se mostrando atenta). Na sala de aula, ambas tendem a obedecer às ordens sem questionar (item 45), o que pode ocorrer devido ao ambiente mais contido e controlado da sala de aula.

\section{Influência do contexto escolar no perfil de competência social da criança com DT e da criança com AU}

Em relação à influência do contexto no perfil de interação da criança com DT, os resultados demonstram que esse se manteve constante nos dois ambientes na maioria das dimensões da escala. Desse modo, parece que o contexto não exerce muita influência no comportamento social da criança com DT, tal como ocorre com a criança com AU. Entretanto, naqueles itens em que houve diferenças pode-se perceber que a criança com DT também apresentou comportamentos de Sociabilidade/Cooperação mais frequentes, no pátio (itens 9, 21 e 42). Da mesma forma que a criança com AU, a criança com DT mostrou-se mais dependente na sala de aula que no pátio (itens 29, 30, 34, 35 e 45). Devido às diferenças de atividades, área e densidade de crianças, a influência positiva e favorecedora do pátio nas interações de crianças pré-escolares parece ser uma tendência geral, não sendo diferente para crianças com transtornos no desenvolvimento. Estudos realizados com base na perspectiva da psicologia ambiental (Frost, Shin, \& Jacobs, 1998; Sager, Sperb, Roazzi, \& Martins, 2003) demonstraram que em ambientes maiores as crianças apresentaram mais interações associativas e paralelas do que em menores (como pátios pequenos ou sala de aula), onde demonstraram maiores níveis de irritação e frustração.

$\mathrm{Na}$ análise da influência do contexto escolar no perfil de competência social da criança com AU, verificou-se que a maioria dos comportamentos descritos nos itens que compõem a escala foi semelhante nos dois contextos. Isso demonstra a consistência das características identificadas tanto naqueles itens indicativos de desorganização do self (Tabela 1) e dependência (Tabela 2) típicos do autismo, quanto daqueles que demonstram frequentes comportamentos de sociabilidade/ cooperação, asserção social (Tabela 1) e baixa frequência de comportamentos agressivos (Tabela2). 
Desse modo, os indícios de competência social da criança AU persistem, independentes do contexto. Trata-se de mais uma evidência que vêm contra o estereótipo da criança com autismo como totalmente alheia e incapaz de estabelecer relações sociais.

Entretanto, em alguns itens o comportamento da criança com AU foi diferente em relação ao contexto, os quais devem ser considerados ao se analisar a influência do ambiente no seu comportamento social. As análises demonstram que o contexto que mais favoreceu a expressão da competência social da criança com AU foi o pátio. É possível perceber que, nesse contexto, a criança, ainda que com pouca frequência, foi mais solicitada pelo grupo (item 3), agiu com mais independência e autonomia (item 6) e melhor expressou suas intenções de modo claro e direto (item 37). Além disso, a criança com $\mathrm{AU}$ foi menos frequentemente rejeitada pelo grupo (item 16) no pátio do que na sala de aula, indicando maior aceitação pelos pares neste contexto. Como consequência, comportamentos de reagir mal quando contrariada (item 25), ou comportamentos de irritação (item 46) não foram observados. As diferenças dos itens também apontaram que a criança com AU foi mais impulsiva (item14) e menos disposta a ouvir os outros e negociar (item 18) na sala de aula. Observa-se ainda que nos itens considerados semelhantes com diferença de um ponto, há uma tendência geral de o escore ser um ponto mais alto, no pátio, naqueles itens que se referem à Sociabilidade/Cooperação (itens $1 \mathrm{e}$ 42) e Asserção Social (itens 36 e 40) e um ponto mais alto na sala de aula, naqueles referentes à Dependência (itens 8 , 22,30 e 38). Isso demonstra que as atividades realizadas no pátio têm proporcionado melhor integração da criança com AU com o grupo e vice-versa e o desenvolvimento de comportamentos mais adaptativos.

Uma possível explicação para a redução da competência social em sala de aula pode ser o caráter das atividades desenvolvidas nesse ambiente que tendem a exigir uma maior compreensão da linguagem e do pensamento simbólico. Essa dificuldade na comunicação e na simbolização é um marcador do autismo. Neste sentido, atividades que exigem habilidades simbólicas como desenhos, jogos de construção, leituras, etc. são de difícil compreensão para a criança com AU, a qual pode se tornar mais irritável, dispersa, desatenta e até agressiva como protesto pela dificuldade em acompanhar as tarefas.

O estudo desenvolvido por Kok, Kong e Bernard- Opitz (2002) encontrou resultados semelhantes quanto aos contextos que favorecem a interação com pares. Os autores compararam o efeito de abordagens de jogo facilitado (várias atividades para a criança escolher e fazer com seus pares) e estruturado (atividade com pares instruída pelo experimentador) na comunicação e comportamento de jogo de crianças pré-escolares com AU. Foi observada alta frequência de jogo inapropriado e a falta de iniciações comunicativas na condição de jogo estruturado comparado ao jogo facilitado, também atribuído à natureza das atividades oferecidas. Diante disso, a falta de foco nas atividades, menor frequência de interações e maior rejeição do grupo podem consequentemente ocorrer dificultando a inclusão da criança com AU na sala de aula.

Diante do desafio em participar de atividades que requerem habilidade representacional (simbólica), observa-se a preferência da criança em observar a queda de objetos, empilhar ou enfileirar blocos ou apenas folhear livros. Estas ações são sensoriais e possuem uma natureza repetitiva, tornando-se, portanto, mais atrativas e compreensíveis às crianças com AU. Elas melhor respondem a estímulos visuais e sinestésicos devido a dificuldades nos processos de pensamento simbólico e abstrato. Todavia, essa preferência da criança com AU não deve ser compreendida como um isolamento proposital, mas sim como a dificuldade em compreender a forma usual de interpretar e corresponder ao que lhe é solicitado. A partir disso, é também possível compreender a preferência dessas crianças por brincadeiras de "pega-pega" no pátio, ao invés de brincadeiras de "esconde-esconde". Esta última é mais sofisticada do ponto de vista cognitivo, exigindo maior entendimento das regras e objetivos da brincadeira. Obviamente isso não significa que atividades de cunho simbólico não devam ser apresentadas às crianças com $\mathrm{AU}$, pois o trabalho pedagógico integrador deve adaptar-se para transformar as limitações.

Apesar de ter sido verificado baixa iniciativa da criança com AU para procurar os colegas (item 1) ou colaborar com a turma (item 2) na sala de aula, houve resposta quando requisitada pelo grupo (item 7), ou seja, ela interage quando solicitada. Esse dado é também corroborado pelo estudo de Kok, Kong e Bernard-Opitz (2002), cujos resultados indicaram que respostas comunicativas podem ser mais frequentes sob condições de jogo estruturado. Estudos desenvolvidos por Zercher, Hunt, Schuler e Webmaster (2001) e Wolfberg e Schuler (1993) também indicaram que a participação de crianças com AU em jogos integrados com crianças com DT, programados e estruturados de acordo com o seu nível de desenvolvimento, produziu aumento significativo na atenção compartilhada de objetos, jogo simbólico e expressão verbal. Neste sentido, considerar as particularidades da criança com AU é fundamental para que as atividades propostas em qualquer ambiente alcancem seus objetivos. Diante disso, é possível novamente perceber o papel fundamental que as interações com pares desempenham no desenvolvimento de diversas habilidades sociais e cognitivas (Almeida, 1997; Dessen \& Aranha, 1994; Hartup, 1996, Schaffer, 1996), bem como a importância dessa convivência para as crianças com AU no contexto escolar, através da inclusão.

Outra possível explicação para a maior frequência de comportamentos de impulsividade (item 14), irritabilidade (item 46) e reações negativas (itens 18 e 25) na sala de aula pode ser o excesso de estimulação social nesse contexto (barulhos e conversas em um pequeno espaço). Dawson e Lewy (1989), com base em seu modelo de hipersensibilidade sensorial, colocam que as interações sociais com crianças com AU podem ser dificultadas por um aspecto central: o ser humano é uma fonte rica em estímulo (visual, auditivo e tátil), cujo impacto na criança com AU pode ser a sobrecarga sensorial. As "pausas" na interação, muitas vezes percebidas como uma "rejeição" à aproximação do outro, seriam uma forma de se restabelecer dessa sobrecarga de estimulação. De fato, comportamento similar pode ser observado em bebês com desenvolvimento típico, principalmente no primeiro semestre de vida, durante interações sociais (Trevarthen, 2000). 


\section{Considerações Finais}

Houve evidência de competência social na criança com $\mathrm{AU}$, ao participar de atividades em grupo, ser solicitada pelos colegas, aceitar sugestões e pedidos dos outros, mostrar-se amigável, afetiva e disposta. Chama a atenção de que, nos aspectos da competência social em que ela diferiu da criança com DT, essa diferença decorre mais em função da menor frequência/intensidade dos comportamentos sociais do que da sua ausência per se. É ainda interessante notar que alguns comportamentos relacionados à Agressão e Desorganização do Self não foram observados na criança com AU e a criança com DT também demonstrou alguns comportamentos marcadamente dependentes em relação ao grupo, bem como de agressão e desorganização.

Sobre a influência do contexto escolar, a expressão da competência social da criança com AU na sala de aula, comparada ao pátio, foi mais dificultada do que a da criança com DT. Entretanto, há evidências de que isso também tende a ocorrer com as crianças de DT (Frost, Shin, \& Jacobs, 1998).

A utilização da metodologia Q-sort, com base em um estudo de caso, permitiu identificar dificuldades já esperadas por serem inerentes à síndrome, mas também demonstrar o quanto os comportamentos da criança com AU foram semelhantes aos de sua colega em alguns aspectos. Entretanto, ressalta-se que os resultados obtidos aplicam-se a estas crianças e investigações com amostras mais amplas são necessárias para condiderar a generalidade destes achados, sobretudo quando consideramos a extensa variação na expressão dos sintomas do autismo. Estudos envolvendo crianças com DT de outra faixa etária e nível de desenvolvimento ou crianças com AU mais comprometidas, talvez resultassem em achados mais discrepantes.

O estudo de caso utilizando a metodologia Q-sort e o avaliador "cego" na observação sistemática mostrou-se adequado ao propósito do estudo ao fornecer dados objetivos, sobretudo na produção de evidências de similaridades entre as duas crianças. No entanto, verificou-se que alguns itens da escala ainda necessitam de refinamento em sua definição operacional.

Quanto às implicações desses resultados para a prática educativa de crianças com AU em situação de inclusão, há também algumas considerações. As diferenças observadas entre os contextos em alguns comportamentos da criança com AU, demonstram a necessidade da implementação de práticas pedagógicas que levem em conta as dificuldades da criança. Neste estudo, verificou-se que as atividades que requerem altos níveis de simbolização (ex. desenhos e contos infantis) tendem a desencadear reações mais desadaptativas. Portanto, sugere-se que nestas situações sejam oferecidas atividades alternativas para as crianças (ex. jogos de encaixe, manipulação de livros). Estas atividades, sendo de menor demanda simbólica, permitem o maior envolvimento da criança com autismo com as outras crianças na sala de aula. Além disso, faz-se necessário respeitar alguns momentos de "isolamento" da criança. Ela necessita destas "pausas" na interação para se restabelecer da sobrecarga de estímulos e da demanda social que naturalmente é maior no ambiente de sala de aula, devido à área reduzida, à proximidade entre as crianças e à natureza das atividades. Antecipar ações e o anúncio de mudanças de rotina, atividades ou contextos podem ser úteis para evitar a desorganização do comportamento frente ao desconhecido. Portanto, é de fundamental importância o conhecimento acerca das particularidades no desenvolvimento destes indivíduos e o nível evolutivo da criança para que estratégias e ações pedagógicas respeitem e potencializem suas habilidades, garantindo a efetiva inclusão das crianças com AU. A partir da constatação de que o pátio melhor favorece a expressão da competência social da criança com AU, aponta-se a necessidade de estudos que investiguem mais especificamente quais os fatores desse contexto que melhor promovem a interação com pares. Da mesma forma, novos estudos são necessários para identificar fatores que favorecem a competência social na sala de aula e o papel do professor nesse processo, especialmente em pesquisas com delineamentos longitudinais.

As evidências de competência social na criança com AU são de extrema importância na desconstrução dos mitos que cercam essas crianças. $\mathrm{O}$ retrato de um indivíduo totalmente ausente e vivendo em um mundo à parte não se mantém quando se consideram os dados empíricos deste e de outros estudos. Desse modo, pode-se pensar que a inclusão escolar de crianças com AU é algo possível, desde que fundamentada no conhecimento, garantindo os recursos necessários e a clareza acerca do papel da escola.

\section{Referências}

Almeida, A. (1997). As relações entre pares em idade escolar. Um estudo de avaliação da competência social pelo método Q-sort. Tese de Doutorado, Universidade do Minho, Portugal.

Anderson, A., Moore, D. W., Godfre, R., \& Fletcher-Flinn, C. M. (2004). Social skills assessment of children with autism in free-play situations. Autism, 8, 369-385.

Associação Psiquiátrica Americana (2002). Manual diagnóstico e estatístico de transtornos mentais. Porto Alegre: Artes Médicas.

Avila, C. F., Tachibana, M., \& Vaisberg, T. M. J. A. (2008). Qual é o lugar do aluno com deficiência? O imaginário coletivo de professores sobre a inclusão escolar. Paidéia, 18 (39), 155-164.

Baptista, C. R. (2002). Integração e autismo: Análise de um percurso integrado. In C. R. Baptista, \& C. A. Bosa, (Eds.), Autismo e educação: Reflexões e propostas de intervenção (pp. 127-139). Porto Alegre: Artmed.

Baptista, C. R., Vasques, C. K., \& Rublescki (2003). Educação e transtornos globais do desenvolvimento: Em busca de possibilidades. Cadernos da APPOA, 114, 31-36.

Barbosa, M. C. (2007). Culturas escolares, culturas de infância e culturas familiares: As socializações e a escolarização no entretecer destas culturas. Educação e Sociedade, 28, 10591083.

Beyer, H. O. (2005). Inclusão e avaliação na escola de alunos com necessidades educacionais especiais. Porto Alegre: Meditação.

Bosa, C. A. (2002). Autismo: Atuais interpretações para antigas observações. In C. R. Baptista, \& C. Bosa, (Eds.), Autismo e educação: Reflexões e propostas de intervenção (pp. 21-39). Porto Alegre: Artmed.

Bosa, C. A. (2006). Autismo: Intervenções psicoeducacionais. Revista Brasileira de Psiquiatria, 28, 47-53. 
Brasil (2001). Estatuto da criança e do adolescente. São Paulo: Conselho Estadual dos Direitos da Criança e do Adolescente de São Paulo.

Camargo, S. P. H., \& Bosa, C. A. (2009). Competência social, inclusão escolar e autismo: Revisão crítica da literatura. Psicologia e Sociedade, 21(1), 65-74.

Castro, R. E. F., Melo, M. H. S., \& Silvares, E. F. M. (2003). O julgamento de pares de crianças com dificuldades interativas após um modelo ampliado de intervenção. Psicologia: Reflexão e Crítica, 16, 309-318.

Chamberlain, B. O. (2002). Isolation or involvement? The social networks of children with Autism included in regular classes. Dissertation Abstracts International, 62(8-A), 2680 (UMI No.AAI3024149).

Chen, X., \& French, D.C. (2008). Children's social competence in cultural context. Annual Review of Psychology, 59(1), 591-616.

Corsaro, W. A. (1997). The Sociology of childhood. Pine Forge Press. Thousand Oaks.

Dawson, G., \& Lewy, A. (1989). Arousal, attention, and socioemotional impairments of individuals with autism. In G Dawson (Ed.), Autism: New perspectives on nature, diagnosis, and treatment (pp. 3-21). New York: Guilford Press.

Del Prette, Z. A. P., \& Del Prette, A. (2006). Avaliação multimodal de habilidades sociais em crianças: Procedimentos, instrumentos e indicadores. In M. Bandeira, Z. A. P. Del Prette \& A. Del Prette (Eds.), Estudos sobre habilidades sociais e relacionamento interpessoal (pp. 47-68), São Paulo: Casa do Psicólogo.

Dessen, M. A., \& Aranha, M. S. L. F. (1994). Padrões de interação social nos contextos familiar e escolar: Análise e reflexões sob a perspectiva do desenvolvimento. Temas em Psicologia, $3,73-90$.

Facion, J. R., Marinho, V., \& Rabelo, L. (2002). Transtorno do autista. In J.R. Faciom, (Ed.), Transtornos invasivos do desenvolvimento associados a graves problemas de comportamento: reflexões sobre um modelo integrativo (pp. 23-28). Brasília: CORDE.

Frederickson, N.; Warren, L., \& Turner, J. (2005). Circle of friends - An exploration of impact over time. Educational Psychology in Practice, 21, 197-217.

Frost, J. L., Shin, D., \& Jacobs, P. J. (1998). Physical environments and children's play. In O. N. Saracho \& B. Spodek (Eds.), Multiple perspectives on play in early childhood education (pp. 255-294). Albany: State University of New York.

Girolametto, L., \& Weitzman, E. (2007). Promoting peer interaction skills. Topics in Language Disorders, 27(2), 93-110.

Goldberg, K. (2002). A percepção do professor acerca do seu trabalho com crianças portadoras de autismo e síndrome de Down: Um estudo comparativo. Dissertação de Mestrado, Universidade Federal do Rio Grande do Sul, Porto Alegre.

Guaralnick, M. J., Connor, R. T., Hammond, M. A., Gottman, J. M., \& Kinnish, K. (1996). The peer relations of preschool children with communication disorders. Child Development, 67, 471-489.

Hartup, W. W. (1992). Friendships and their developmental significance. In H. McGurk (Ed.), Childhood social development (pp. 175-205). Gove: Erlbaum.

Hartup, W. W. (1996). The company they keep: Friendships and their developmental significance. Child Development, 67, 1-13.
Hay, D., Payne, A., \& Chadwick, A. (2004). Peer relations in childhood. Journal of Child Psychology and Psychiatry, 45, $84-108$

Kanner (1943). Affective disturbances of affective contact. The Nervous Child, 2, 217 - 250.

Karagiannis, A., Stainback, S., \& Stainback, W. (1999). Fundamentos do Ensino Inclusivo. In S. Stainback \& W. Stainback (Eds.), Inclusão - Um guia para Educadores (pp. 21-34). Trans. M. Lopes. Porto Alegre: Artmed.

Kauffman, J. M., Landrum, T. J., Mock, D. R., Sayeski, B., \& Sayeski, K.L. (2005). Diverse knowledge and skills require a diversity of instructional groups. Remedial and Special Education, 26(1), 2-6.

Klin, A. (2006). Autismo e síndrome de Asperger: Uma visão geral. Revista Brasileira de Psiquiatria, 28, 3 - 11.

Kok, A. J.; Kong, T. Y., \& Bernard- Opitz, V. (2002). A comparison of the effects of structured play and facilitated play approaches on preschoolers with autism: A case study. Autism, 6, 181-196.

Kristen, R.; Brandt, C., \& Connie, K. (2003). General education teachers' relationships with included students with Autism. Journal of Autism and developmental disorders, 33, 123-130.

Lira, S. M. de (2004). Escolarização de alunos com transtorno autista: histórias de sala de aula. Dissertção de Mestrado, Universidade do Estado do Rio de Janeiro, Rio de Janeiro.

Lordelo, E. R., \& Carvalho, A. M. A. (1998). Creche como contexto de desenvolvimento: Parcerias adulto-criança e criançacriança. Temas de Psicologia, Ribeirão Preto, 6, 117-128.

Mantoan, M. T. E. (1998). Integração x Inclusão - Educação para todos. Pátio, 5, 48-51.

Mathieson, K., \& Banerjee, R. (2010). Pre-school peer play: The beginnings of social competence. Educational and Child Psychology, 27(1), 9-20.

Ochs, E., Kremer-Sadlik, T., Solomon, O., \& Sirota, K. G. (2001) Inclusion as social practice: Views of children with Autism. Social Development, 10, 399 - 419.

Oliveira, Z. M. R., \& Rossetti-Ferreira, M. C. (1993). O valor da interação criança-criança em creches no desenvolvimento infantil. Cadernos de Pesquisa, 87, 62-70.

Pepler, D. J., \& Craig, W. M. (1998). Assessing children's peer relationships. Child Psychology \& Psychiatry Review, 3, $176-182$.

Pereira, G. M. A. A. (2003). Autismo e inclusão: Uma proposta de programa educacional. Dissertação de Mestrado, Pontifícia Universidade Católica do Rio de Janeiro, Rio de Janeiro.

Rubin, K., Coplan, R., \& Bowker, J. (2009). Social withdrawal in childhood. Annual Review of Psychology, 60(1), 141-171.

Sager, F., Sperb, T. M., Roazzi, A., \& Martins, F. M. (2003). Avaliação da interação de crianças em pátios de escolas infantis: Uma abordagem da psicologia ambiental. Psicologia Reflexão e Crítica, 16, 203 - 215.

Sant'Ana, I. M. (2005). Educação inclusiva: Concepções de professores e diretores. Psicologia em Estudo, 10, 227 - 234.

Sassaki, R. K. (1998). Integração e Inclusão: Do que estamos falando? Temas sobre desenvolvimento, 39, 45 - 47.

Serra, D. C. G. (2004). A inclusão de uma criança com Autismo na escola regular: Desafios e processos. Dissertação de Mestrado, Universidade do Estado do Rio de Janeiro, Rio de Janeiro.

Schaffer, H. R. (1996). Social development. Cambridge: Blackwell Publisher. 
Trevarthen, C. (2000). Intrinsic motives for companionship in understanding: Their origin, development, and significance for infant mental health. Infant Mental Health Journal, 22, 95-131.

Waters, E., \& Sroufe, L. A. (1983). Social competence as a developmental construct. Developmental Review, 3, 79 - 97.

Williams, S., Ontai, L., \& Mastergeorge, A. (2010). The development of peer interaction in infancy: Exploring the dyadic processes. Social Development, 19(2), 348-368.

Wolfberg, P. J., \& Schuler, A. L. (1993). Integrated play groups: A model for promoting the social and cognitive dimensions of play in children with autism. Journal of Autism and Developmental Disorders, 23, 467-489.

Worrell, J. (2008). How secondary schools can avoid the seven deadly school "sins" of inclusion. American Secondary Education, 36(2), 43-56.

Yang, T. R., Wolfberg, P. J., Wu, S. C., \& Hwu, P. Y. (2003). Supporting children on the autism spectrum in peer play at home and school: Piloting the integrated play groups model in Taiwan. Autism, 7, $437-453$.
Zercher, C., Hunt, P., Schuler, A., \& Webmaster, J. (2001). Increasing joint attention, play and language trough peer supported play. Autism, 5, 374-398.

Zilmer P. (2003). Reflexões sobre a prática: Escola ou clínica? In M. S Charczuk \& M. N. Folberg (Eds.), Crianças psicóticas e autistas: A construção de uma escola (pp. 27-38). Porto Alegre: Mediação.
Recebido em 12.01.2010

Primeira decisão editorial em 19.08.2010

Versão final em 16.03.2012

Aceito em 21.06.2012 